\title{
Ectoparasitic community of the Mahali mole-rat, Cryptomys hottentotus mahali: potential host for vectors of medical importance in South Africa
}

\author{
Dina M. Fagir ${ }^{1^{*}} \mathbb{D}$, Nigel C. Bennett ${ }^{1 \dagger}$, Eddie A. Ueckermann², Alexandra Howard ${ }^{1}$ and Daniel W. Hart ${ }^{1 \dagger}$
}

\begin{abstract}
Background: The endemic rodent family of Bathyergidae in Africa, particularly South Africa, are understudied as reservoirs of diseases of significant medical importance. Considering the diversity and wide distribution of African mole-rats in South Africa, many of these bathyergids could act as carriers of zoonoses.
\end{abstract}

Methods: The present study assessed the ectoparasite community of the Mahali mole-rat (Cryptomys hottentotus mahali). We aimed to identify possible parasitic arthropods that may infest this mole-rat species and explore host preference, contributions of seasonality, host sex and body mass as well as social class and colony size on ectoparasite assemblage prevalence and abundance.

Results: A limited number of ectoparasite species were found on C. h. mahali belonging to two significant taxa: mites (Acari) and fleas, with mites being the most prevalent and abundant. We recorded the presence of $X$. philoxera, a flea well known as the principal reservoir of plague in the southern African region on the Mahali mole-rats. Only three mite species were collected: Androlaelaps scapularis, Androlaelaps capensis and Laelaps liberiensis. Seasonal peaks in prevalence and abundance of $X$. philoxera and A. scapularis were observed during summer. Xenopsylla philoxera abundance and $A$. scapularis loads significantly increased on reproductive mole-rat individuals in comparison to nonreproductive individuals.

Conclusion: Despite the wide distribution of the subterranean African mole-rats, studies investigating their parasitic fauna remain limited and scarce. This dearth in knowledge raises the concern regarding their potential role as an endemic reservoir for zoonotic diseases. Consequently, additional sampling of their ectoparasitic community throughout their distributional range and research addressing their role as a reservoir for zoonotic diseases in southern Africa are urgently needed.

Keywords: Cryptomys, Mole-rats, Ectoparasites, Seasonality, Fleas, Xenopsylla, Androlaelapid mites, Zoonotic diseases

${ }^{*}$ Correspondence: dmfagir@zoology.up.ac.za

${ }^{\dagger}$ Nigel C. Bennett and Daniel W. Hart contributed equally to this work

${ }^{1}$ Department of Zoology and Entomology, University of Pretoria, Private Bag X20, Hatfield 0028, South Africa

Full list of author information is available at the end of the article

\section{Background}

Members of the Order Rodentia globally harbour different ectoparasitic arthropods, of which many are vectors for diseases of medical and veterinary importance [1]. Factors driving parasite prevalence and abundance patterns may be divided into two general categories, environmental factors (abiotic) and host-related factors (biotic) $[2,3]$. The differences in parasite burdens may

c) The Author(s) 2021. This article is licensed under a Creative Commons Attribution 4.0 International License, which permits use, sharing, adaptation, distribution and reproduction in any medium or format, as long as you give appropriate credit to the original author(s) and the source, provide a link to the Creative Commons licence, and indicate if changes were made. The images or other third party material in this article are included in the article's Creative Commons licence, unless indicated otherwise in a credit line to the material. If material is not included in the article's Creative Commons licence and your intended use is not permitted by statutory regulation or exceeds the permitted use, you will need to obtain permission directly from the copyright holder. To view a copy of this licence, visit http://creativecommons.org/licenses/by/4.0/. The Creative Commons Public Domain Dedication waiver (http://creativecommons.org/publicdomain/zero/1.0/) applies to the data made available in this article, unless otherwise stated in a credit line to the data. 
result from a number of these factors (i.e. abiotic and biotic) that are not always mutually exclusive, and their contributions may be difficult to disentangle $[2,4]$. Seasonal patterns can be linked to the parasite life-cycle and how, or to what degree, changes in temperature, rainfall and humidity may affect and/or determine the duration of the developmental stages (i.e. eggs, larva/nymph, adult) in these parasites, as well as the periods, spent off-host by a particular ectoparasite [5-7]. Parasite burdens often show a male-biased pattern with males being infested by more ectoparasites than females $[8,9]$. Males tend to be the larger of the sexes in the majority of mammal species and to be able to sustain higher parasite numbers as they represent more substantial resources [8]. The immunosuppressive properties of testosterone, associated with breeding, could also be a contributing factor to higher male infestation [9-12]. Another host-related factor affecting parasite burdens is linked to the degree of sociality [13]. Many studies have reported an increase in parasite burdens with the increases in group size [14, 15]. In contrast, other studies failed to support this and did not find a correlation between parasite burdens and group size [16]. A study by Bordes et al. [17] suggested that a decrease in ectoparasite loads might be affected by different factors such as the host's environment (e.g. temperature, rainfall, number of potential hosts and the number of other ectoparasites) as well as increased host defence strategies against ectoparasite transmission in social species [17-19]. Further research is required to disentangle the abiotic and biotic factors affecting differences in parasite burdens; this is particularly true in social mammalian species.

African mole-rats (family Bathyergidae) are subterranean hystricomorph rodents with a wide distributional range throughout sub-Saharan Africa [20]. Many African mole-rat species are deemed pests since they eat the roots of crops and cause property damage due to their tunnel systems. The Mahali mole-rat, Cryptomys hottentotus mahali, lives in colonies with only one reproductive female (queen) and up to three potential reproductive males [20]. The remaining members of the colony are reproductively inactive, with decreased levels of reproductive hormones such as testosterone and perform alloparental care to the queen's offspring [21, 22]. The members in the colony exhibit allogrooming, which could reduce ectoparasite loads, and the movement of animals is restricted mainly by rainfall $[20,23]$. A large number of studies have focused on the social system and reproductive physiology of mole-rats [21, 22, 24-26]. However, in the southern African region, particularly South Africa, very few studies have been carried out on parasites of bathyergids with only a limited number of hosts [2, 27-31]. Thus, we aimed to address this dearth of knowledge and supply an inventory list of the ectoparasites of the aseasonal breeding Mahali mole-rat. Also, the present study aimed to assess the contributions of host sex, social class, group size and season on the ectoparasitic community in the cooperatively breeding Mahali mole-rat.

\section{Methods}

The Mahali mole-rat (Cryptomys hottentotus mahali) was captured on smallholdings and farms in and around the area of Patryshoek, Pretoria $\left(25^{\circ} 40^{\prime} \mathrm{S}, 28^{\circ} 2^{\prime} \mathrm{E}\right)$, South Africa. The region of Patryshoek comprises bushveld of South Africa, characterised by cold, dry winters and hot moist summers (South African Weather Bureau). Mahali mole-rats were caught every month between October 2016 and September 2017. A total of 31 colonies were captured using Hickman live-traps baited with sweet potatoes. Traps were placed at the entrance of excavated burrows each month during the capture period and were checked every $2 \mathrm{~h}$ from dusk till dawn. Colonies were caught in their entirety, and a colony was deemed to be entirely trapped if no trap activity was observed for 5 consecutive days. Once captured, colonies were transported back to the mole-rat laboratory at the University of Pretoria, Department of Zoology and Entomology. Captured animals were housed with their colony mates in plastic crates $(49.5 \times 28 \mathrm{~cm})$ with wood shavings and paper towelling provided as nesting material. The molerats were fed fresh sweet potato and apples daily until assessed for ectoparasites. All water requirements of the animals were satisfied in the provisioning of food.

In the laboratory, animals were weighed and sexed, and their reproductive status was determined. Reproductive males (RMs) were distinguishable from non-reproductive males (NRMs) by their large descended testes and yellow staining around the mouth. Furthermore, the RM was the heaviest male within each colony, while NRMs were significantly lighter [20]. Reproductive females (queens, RFs) were characterised by having prominent axillary teats and perforated vaginas, which were not observed in the non-reproductive females (NRFs) [32].

Individual animals were examined for ectoparasites within 5 days of capture, and a modified wash technique was applied for the assessment of ectoparasite loads [32]. Mole-rats were anaesthetised (to be used in other studies) with an overdose of isoflurane; subsequently, each individual was washed thoroughly in $100 \mathrm{ml}$ of warm soapy water in a $22 \times 7 \times 5$-cm tub to remove any ectoparasites. The washing process was standardised for all individuals and involved kneading through the fur with the fingertips all the way along and around the body length three times before washing the head and 
the posterior end. Subsequently, individuals were moved back and forth through the water (20 times each side), to remove any ectoparasites that remained caught in the fur. Any additional ectoparasites found on the individual after the process were also collected using tweezers. The wash water was subsequently transferred to a labelled screw-top container until processing. Each wash sample was filtered through a no. 25 US Standard Sieve (710-|j. micron screen) for ectoparasites, and these were then collected and placed into Eppendorf tubes containing $70 \%$ ethanol for preservation. For microscopic examination, mites were cleared and mounted following the techniques described in [33]. Fleas and mites were identified using the morphological keys of [33-35].

Prevalence and mean abundances were calculated for the two higher taxa (i.e. mites and fleas) and the most common individual parasite species. The rarity of some parasite species precluded any meaningful statistical analyses; therefore, only descriptive statistics were reported for the rare parasite species. Data were not normally distributed even after log-transformation (Kolmogorov-Smirnov test: $P<0.0001)$. The effect of the four seasons, i.e. spring (September, October, November 2016, September 2017), summer (December 2016, January, February 2017), autumn (March, April, May 2017), and winter (June, July, August 2017), host sex, host body mass, individual social class (i.e. reproductive and nonreproductive animals) as well as colony size on variation in prevalence and abundance of the ectoparasites were investigated using generalized linear models (GZLMs). A model with a binomial distribution with a logit-link function was selected for prevalence data and a negative-binomial distribution with a log-link function for abundance data. Post hoc analyses were performed with a pairwise comparison using the least significant difference (LSD). All two-way interactions were included in the model, and since none of them yielded a significant result, only main effects were reported in the present study. For meaningful statistical analyses, all different developmental stages of parasite species (i.e. larva, nymph, male, female) were pooled for analysis. We tested the effect of colony size, average temperature and rainfall on ectoparasite burdens in a separate GZLM to avoid parameters overload on the model. All statistical analyses were conducted in IBM SPSS version 25 (IBM SPSS Statistics 25. Ink 2017), and results are reported as the mean \pm standard error (SE).

\section{Results}

A total of 202 individual mole-rats were captured and assessed for ectoparasite loads (Table 1). The overall prevalence of ectoparasites was 69.3\% (140/202 individuals), with a total of 1395 individual ectoparasites (mean abundance $6.91 \pm 1.26$ ) collected from these mole-rats. The ectoparasites collected belonged to two major taxa, fleas and mites, with the androlaelapid mites being the most prevalent and abundant ectoparasites (Table 2). Neither ticks nor lice were found on any mole-rat individuals.

Table 1 Summary of individual Mahali mole-rats (Cryptomys hottentotus mahali, $n=202$ ) captured per season

\begin{tabular}{|c|c|c|c|c|c|}
\hline Season & No. of NRF (\%) & No. of RF (\%) & No. of NRM (\%) & No. of RM (\%) & Total (\%) \\
\hline Spring 2016 & $20(9.9)$ & $3(1.5)$ & $12(5.9)$ & $4(2.0)$ & $39(19.3)$ \\
\hline Summer 2016/2017 & $29(14.4)$ & $6(3.0)$ & $19(9.4)$ & $9(4.5)$ & $63(31.2)$ \\
\hline Autumn 2017 & $10(5.0)$ & $4(2.0)$ & $9(4.5)$ & $5(2.5)$ & $28(13.9)$ \\
\hline Winter 2017 & $21(10.4)$ & $4(2.0)$ & $28(13.9)$ & $7(3.5)$ & $60(29.7)$ \\
\hline Spring 2017 & $4(2.0)$ & $1(0.5)$ & $4(2.0)$ & $3(1.5)$ & $12(5.9)$ \\
\hline Total & $84(41.6)$ & $18(8.9)$ & 72 (35.6) & 28 (13.9) & $202(100)$ \\
\hline
\end{tabular}

$N R F$ non-reproductive female, $R F$ reproductive female, $N R M$ non-reproductive male, $R M$ reproductive male

Table 2 Ectoparasites observed on Mahali mole-rats (Cryptomys hottentotus mahali) in Patryshoek area north of the Magaliesburg mountain, South Africa, over 1 calendar year

\begin{tabular}{|c|c|c|c|c|c|c|c|c|}
\hline Species & $L$ & PN & DN & M & $\mathrm{F}$ & Total & Prevalence (\%) & Mean abundance \pm SD \\
\hline \multicolumn{9}{|l|}{ Fleas } \\
\hline Xenopsylla philoxera & - & - & - & 47 & 37 & 84 & 23.3 & $0.42 \pm 1.000$ \\
\hline \multicolumn{9}{|l|}{ Mites } \\
\hline Androlaelaps scapularis & 1 & 125 & 241 & 113 & 806 & 1286 & 62.4 & $6.37 \pm 17.751$ \\
\hline Androlaelaps capensis & 0 & 0 & 3 & 1 & 18 & 22 & 8.4 & $0.11 \pm 0.397$ \\
\hline Laelaps liberiensis & 0 & 1 & 0 & 2 & 0 & 3 & 1.0 & $0.01 \pm 0.157$ \\
\hline
\end{tabular}

$L$ larva, $P N$ protonymph, $D N$ deutonymph, $M$ male, $F$ female, $S D$ standard deviation 

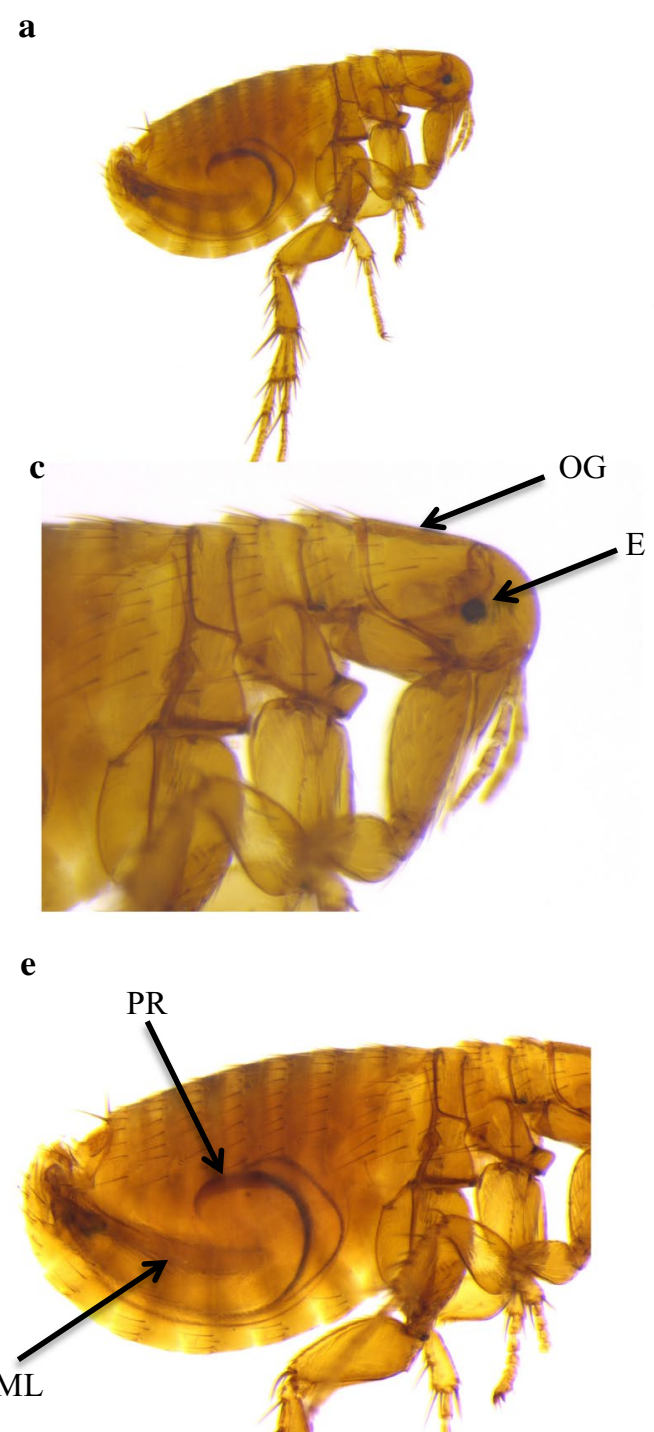

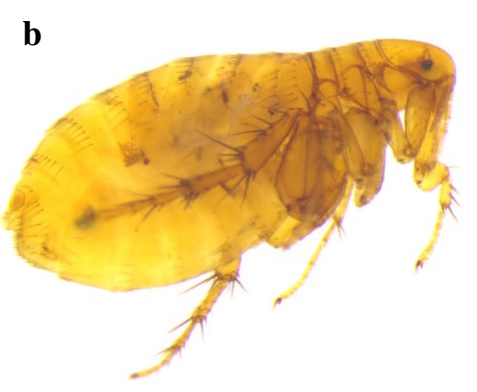

d

$\mathbf{f}$
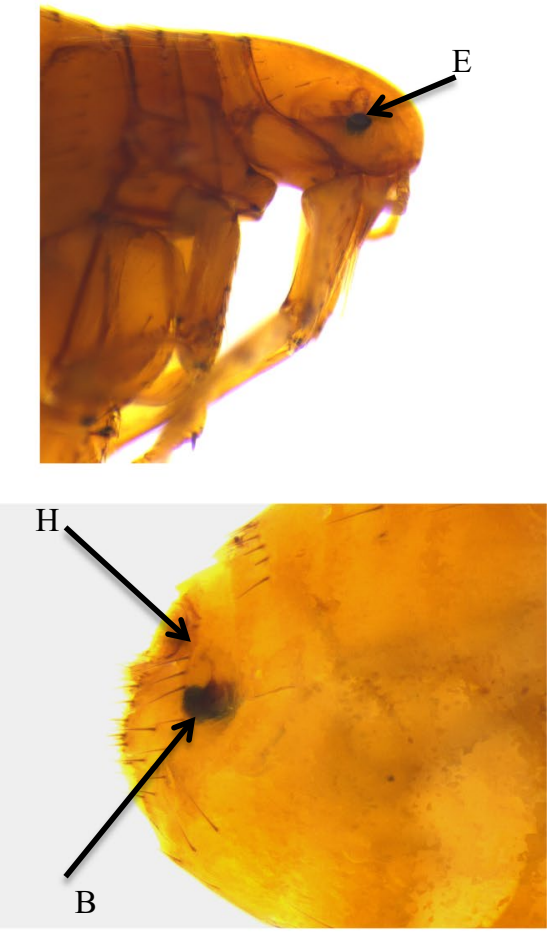

Fig. 1 Xenopsylla philoxera (flea). a Male, whole body. b Female, whole body. c Male, head (note the well-developed and pigmented eye (E) and the shallow occipital groove (OG). $\mathbf{d}$ Female, head (note the well-developed and pigmented eye (E)). e Male, abdomen (phallosome: median lamina $(\mathrm{ML})$ and penis rod (PR). f Female, abdomen (spermatheca: bulga (B) and hilla $(\mathrm{H})$ )

All fleas were identified as Xenopsylla philoxera (Fig. 1). Flea prevalence differed significantly with season, and it was significantly lower in winter $(14.9 \%, n=7)$ compared to spring (29.8\%, $n=14$, LSD: $P \leq 0.043)$ and summer (44.7\%, $n=21$, LSD: $P=0.003)$. Host sex, host body mass, individual status and colony size had no significant effects on flea prevalence (Table 3). Flea abundance was significantly higher in summer (mean $0.63 \pm 0.128$ ) compared to autumn (mean $0.29 \pm 0.115$, LSD: $P=0.042$ ) and winter (mean $0.17 \pm 0.058, P=0.001$ ). In addition, flea abundance was higher in spring (mean $0.50 \pm 0.120$ ) than in winter (mean $0.17 \pm 0.058, P=0.013$ ). Reproductive status of the mole-rat significantly affected the flea abundance, with reproductive males and females harbouring more fleas than non-reproductive ones (Fig. 2). In contrast, sex, body mass and colony size had no significant effect on the abundance of fleas (Table 3).

The mesostigmatic mite, Androlaelaps scapularis, was the most prevalent and abundant species (Table 1, Fig. 3). The majority of $A$. scapularis collected were adults with a bias towards females. Among all individuals captured, A. scapularis prevalence was $62.4 \%$ and did not vary significantly with host sex, nor with the body mass or colony size (Tables 2,3). However, A. scapularis prevalence varied significantly with the seasons and animal status (Tables 2,3). Androlaelaps scapularis 
Table 3 Results of the GLMs for total mite and flea prevalence and abundance of the Mahali mole-rats (Cryptomys hottentotus mahali) over one calendar year

\begin{tabular}{|c|c|c|c|c|c|c|c|}
\hline \multirow[t]{2}{*}{ Parasite } & \multirow[t]{2}{*}{ Factor } & \multicolumn{3}{|c|}{ Prevalence } & \multicolumn{3}{|c|}{ Abundance } \\
\hline & & Wald $x^{2}$ & $d f$ & $P$ & Wald $x^{2}$ & $d f$ & $P$ \\
\hline \multirow[t]{5}{*}{ Xenopsylla philoxera } & Season & 8.215 & 3 & $0.042^{*}$ & 12.513 & 3 & $0.006^{*}$ \\
\hline & Sex & 1.178 & 1 & 0.278 & 0.036 & 1 & 0.849 \\
\hline & Body mass & 0.000 & 186 & 1.000 & 10.719 & 45 & 1.000 \\
\hline & Animal status & 3.218 & 1 & 0.073 & 9.242 & 1 & $0.002^{*}$ \\
\hline & Colony size & 13.592 & 12 & 0.328 & 16.732 & 12 & 0.160 \\
\hline \multirow[t]{5}{*}{ Androlaelaps scapularis } & Season & 15.782 & 3 & $0.001^{*}$ & 146.379 & 3 & $<0.0001^{*}$ \\
\hline & Sex & 0.011 & 1 & 0.916 & 0.181 & 1 & 0.670 \\
\hline & Body mass & 0.000 & 186 & 1.000 & 166.343 & 124 & $0.007^{*}$ \\
\hline & Animal status & 5.364 & 1 & $0.021^{*}$ & 1.862 & 1 & 0.172 \\
\hline & Colony size & 0.000 & 1 & 1.000 & 2.660 & 1 & 0.103 \\
\hline
\end{tabular}

* Significant results

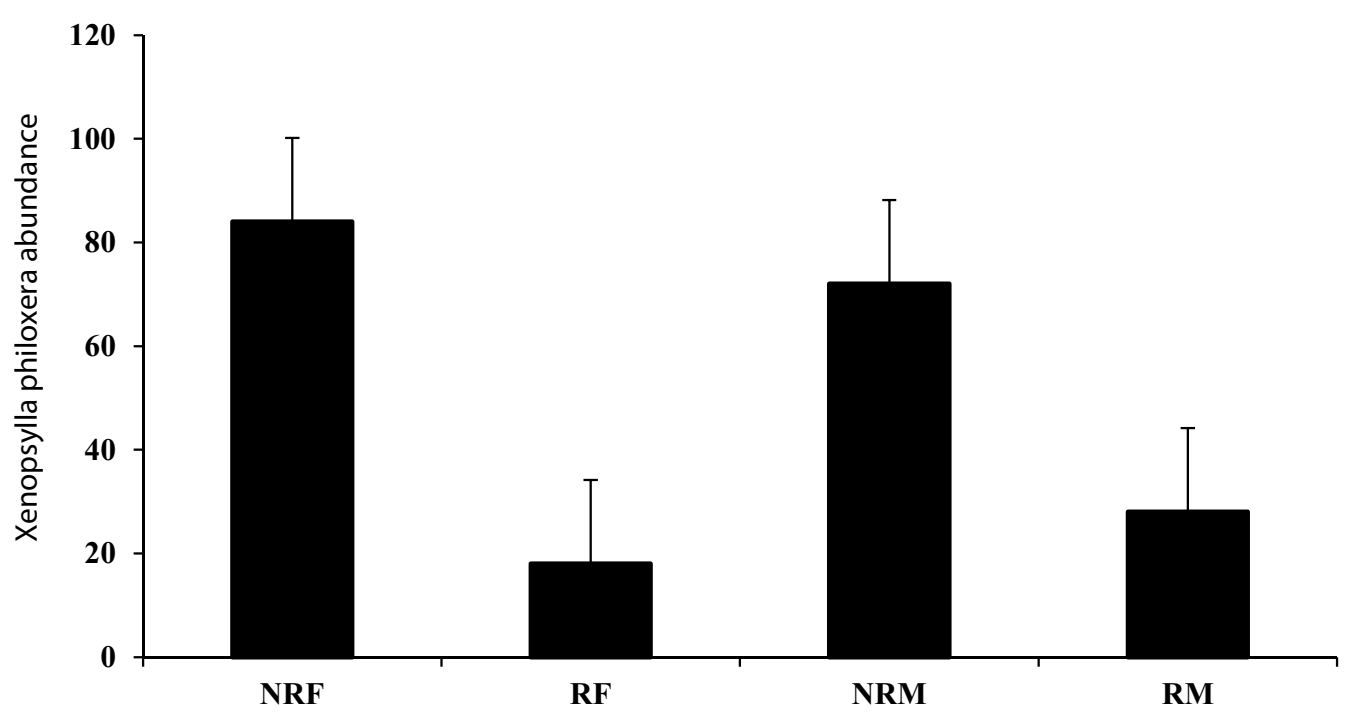

Fig. 2 Effect of host reproductive status on the abundance of Xenopsylla philoxera

prevalence was significantly higher in summer compared to spring, autumn and winter (Fig. 4a, Table 3). Animal reproductive status had a significant effect on $A$. scapularis prevalence (Table 3 ). There was no significant difference between the sexes reproductive status, but the non-reproductive individuals (males and females) were more infested by mites compared to reproductive individuals (Fig. 5, Table 3). Mean abundance of $A$. scapularis was $6.37 \pm 1.249$, and it varied significantly with season (Table 3). Post hoc analyses showed that mite abundance was significantly higher in summer compared to spring, autumn and winter (Fig. 4b). Host body mass had a significant effect on mite abundance; however, post hoc analyses did not confirm these results (LSD: $P \geq 0.731$ ).
In contrast, host sex, reproductive status and colony size had no significant effect on the abundance of $A$. scapularis (Table 3 ).

\section{Discussion}

The parasite assemblage found in the present study was limited to two arthropod taxa (fleas and mites) with a low species diversity. Similar low parasite species diversity has been recorded for several other subterranean rodents $[2,7,20,36]$. The reported low parasite burden may be a result of the subterranean lifestyle of the study species, which could limit exposure to parasites [31, 37-40]. Xenopsylla philoxera has not previously been reported from any South African mole-rats, although another 

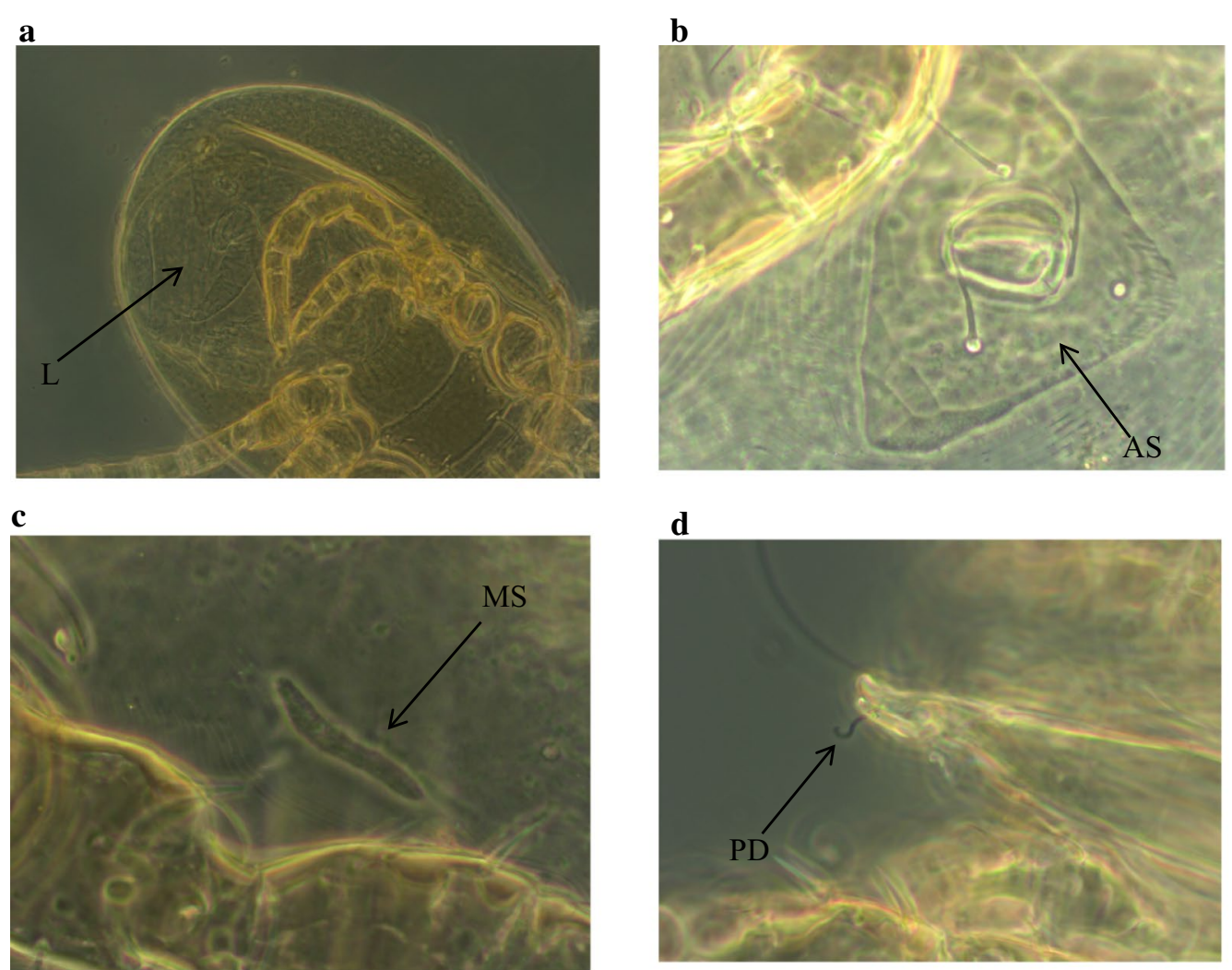

e

f
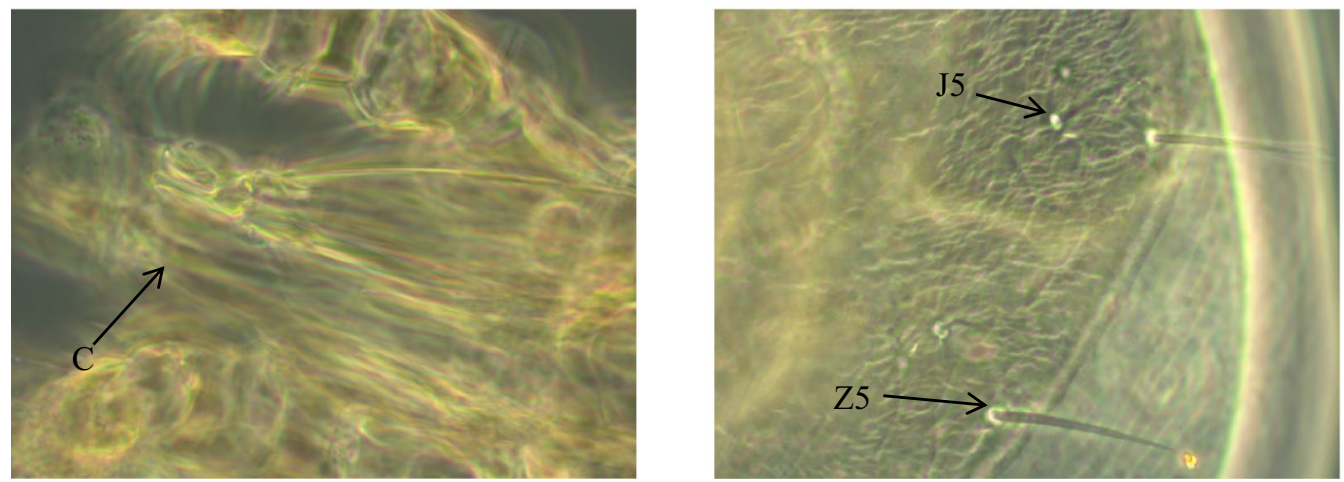

Fig. 3 Female Androlaelaps scapularis (mite). a Opisthogaster with larva (L) inside body (arrow). b Anal shield (AS). c Metapodal shield (MS). d Pilus dentilis (PD). e Chelicera (C). f Dorsal shield showing seta Z5 much longer than seta J5

flea species (Cryptopsylla ingrami) has been reported from $C$. h. hottentotus [37]. The two mites A. scapularis and $A$. capensis have been reported in previous studies from Cryptomys hottentotus hottentotus, C. h. pretoriae and Fukomys damarensis [31, 36, 37, 39]. On the Mahali mole-rat, as well as on closely related species $(C$. h. hottentotus and $C$. $h$. pretoriae), mites were the dominant taxa [31, 37].

Androlaelaps scapularis was observed to be the most prevalent and abundant parasite species, while the prevalence and abundance of $A$. capensis were slightly lower in this present study compared to those of $C . h$. hottentotus [37]. However, the low prevalence and abundance of parasites on Mahali mole-rats are similar to those of $C$. h. pretoriae [31]. Both of these mite species have only ever been recorded from bathyergids, indicating host specificity at the family level [31,35]. Clear seasonal patterns were found for both prevalence and abundances of overall mites and fleas. The burdens of both mites and fleas were higher during the wet summer, 

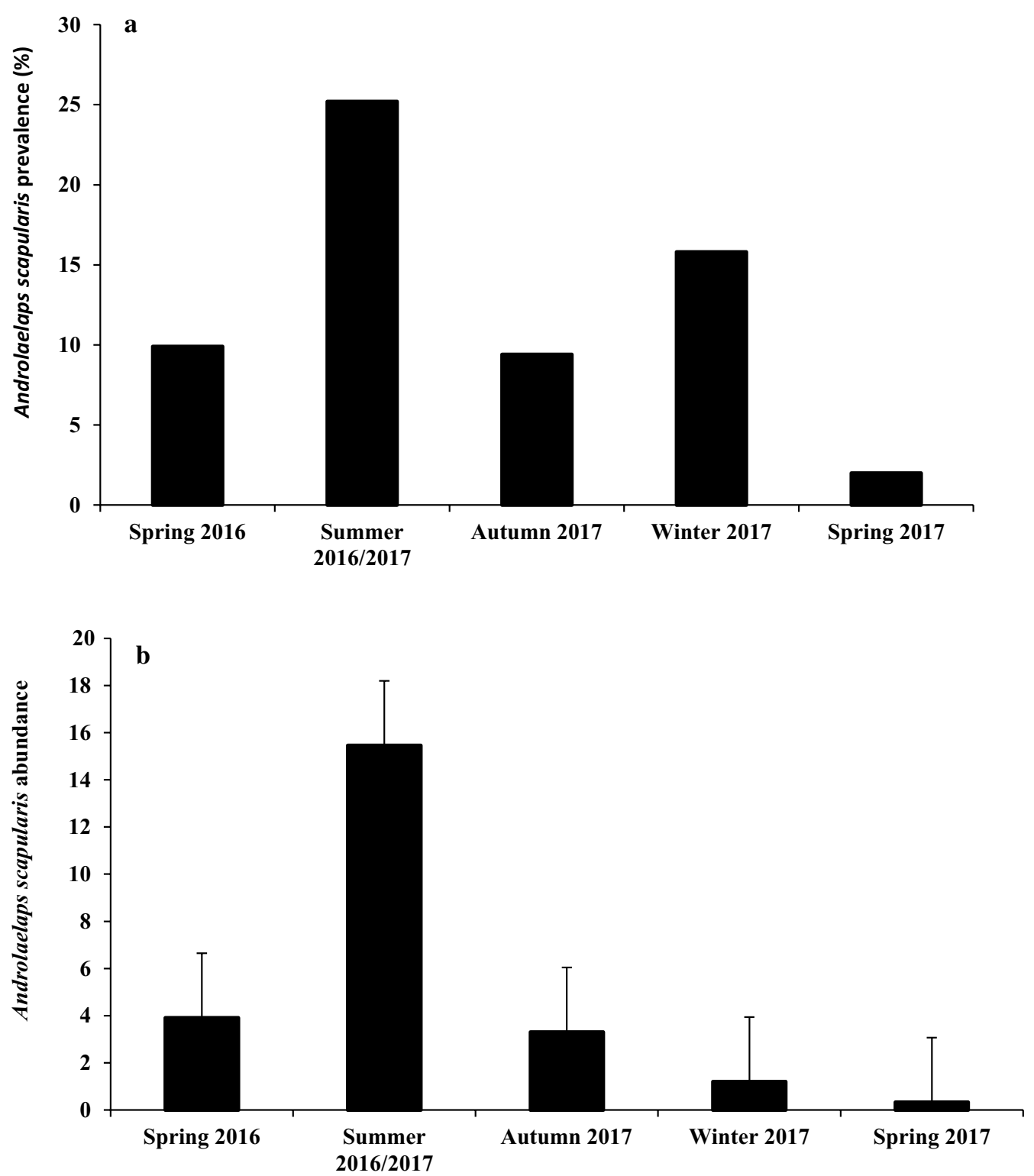

Fig. 4 Seasonal variations in Androlaelaps scapularis on Cryptomys hottentotus mahali in over one calendar year. a Prevalence. b Abundance

as reported for the highveld mole-rat [31]. In contrast, in the common mole-rat (C. h. hottentotus), ectoparasite burdens were higher during the wet winter [37], indicating that changes in humidity rather than temperature are responsible for seasonal variations in ectoparasite burdens. Seasonal variations and the importance of humidity for ectoparasites are well known for various taxa [34]. Seasonal variations in parasite loads may also be a result of a synchronized reproductive cycle between parasite species and their hosts $[34,35]$. The lack of sex bias in the present study may be linked to the sedentary lifestyle of the study species. The same observation has been previously observed in a closely related species $(C$. $h$. pretoriae) [28]. This can be linked to the shared habitat of group members of different sex and reproductive status [31]. Also, activities such as locomotion and dispersal are energetically costly underground; therefore, these activities are often restricted to rainfall seasons when burrow digging is less costly [31]. This results in members of a colony sharing the same burrow system for extended periods; thus, as a result, all colony members have similar parasite exposure regardless of their sex [31]. 


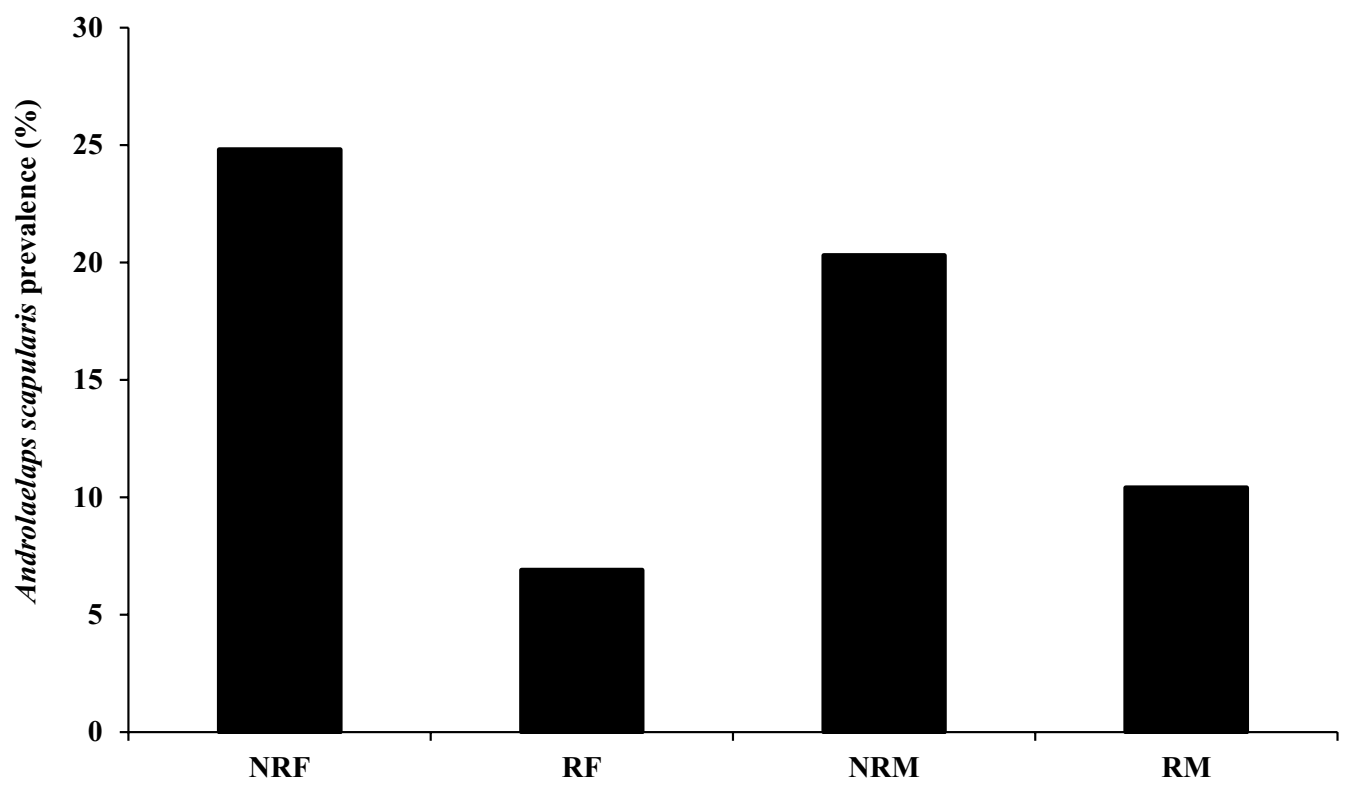

Fig. 5 Effect of host reproductive status on the prevalence of Androlaelaps scapularis

This study, interestingly, revealed that there were reproductive status differences in parasite loads. Reproductive individuals are likely to be older in age, and the lower parasite abundance in these individuals may be an indicator of acquired immunity against parasitic infestation [31]. No significant pattern was found between parasite load and colony size in the study population. Contrastingly, Bordes et al. [18] and Viljoen et al. [31] found a significant relationship between group size and parasite loads. Two possible reasons are put forward to explain the lack of significant differences between parasite abundance and group size in our study. First, the host environment may affect parasite burdens [19]. Host environment refers not only to the abiotic factors, such as temperature and rainfall, but also it extends to some host biotic factors, such as the number of potential hosts and number of other parasite taxa/species, which may affect competition between parasites over space or nutrition [18]. The second hypothesis suggests that social groups may have developed defensive strategies to control parasite transmission, such as increased allogrooming rates in larger groups $[18,19]$.

\section{Conclusions}

In summary, the ectoparasitic fauna of the Mahali molerat was limited to a small number of parasite species and dominated by one mite species. The Androlaelaps mites appear to be specific to the Bathyergidae, whereas the flea appears to be a generalist. Both prevalence and abundance of mite and flea exhibited seasonal peaks in summer.

\section{Abbreviations}

NRF: Non-reproductive female; RF: Reproductive female; NRM: Non-reproductive male; RM: Reproductive male; L: Larva; PN: Protonymph; DN: Deutonymph; M: Male; F: Female; SD: Standard deviation.

\section{Acknowledgements}

We extend our sincere appreciation to the community of Patryshoek for allowing the authors to trap the animals on their property. We also thank the Gauteng Department of Nature Conservation for permits to collect the molerats. We also thank Mr. Dewald Kleynhans for his valuable help with collection and trapping of animals and comments on the manuscript.

\section{Authors' contributions}

Conceived and designed the study: DMF, DWH and NCB. Data collection and parasites counting/identification: DWH, AH, DMF and EAU. Performed statistical analysis: DMF. Contributed materials/analysis tools: NCB, DMF and EAU. Wrote the manuscript: DMF, NCB, DWH, AH and EAU. All authors read and approved the final manuscript.

\section{Funding}

NCB acknowledges funding from the SARChl chair of Mammalian Behavioural Ecology and Physiology from the DST-NRF South Africa, the National Research Foundation (grant no. 64756) and the University of Pretoria, South Africa.

\section{Availability of data and materials}

Data and materials were made available by NCB and DWH.

\section{Ethics approval and consent to participate}

The experimental protocol was evaluated by the Animal Use and Care of the University of Pretoria, ethics clearance number EC044-16.

\section{Consent for publication}

Not applicable.

\section{Competing interests}

The authors declared that they have no competing interests. 


\begin{abstract}
Author details
${ }^{1}$ Department of Zoology and Entomology, University of Pretoria, Private Bag X20, Hatfield 0028, South Africa. ${ }^{2}$ Unit for Environmental Sciences and Management, Potchefstroom Campus, North-West University, Private Bag X6001, Potchefstroom 2520, South Africa.
\end{abstract}

Received: 20 December 2019 Accepted: 10 December 2020 Published online: 06 January 2021

\section{References}

1. Morand S, Krasnov BR. Micromammals and macroparasites from evolutionary ecology to management. Tokyo: Springer; 2006.

2. Poulin R. Evolutionary ecology of parasites: from individuals to communities. Princeton: Princeton University Press; 2007.

3. Shaw DJ, Dobson AP. Patterns of macroparasite abundance and aggregation in wildlife populations: a quantitative review. Parasitology. 1995;111(S1):111-27.

4. Wilson K, Bjørnstad ON, Dobson AP, Merler S, Poglayn G, Randolph SE, et al. Heterogeneities in macroparasite infections: patterns and processes. In: Hudson PJ, Rizzoli A, Grenfell BT, Heesterbeek H, Dobson AP, editors., et al., Ecology of wildlife diseases. New York: Oxford University Press; 2001. p. 6-44.

5. Marshall AG. The ecology of ectoparasitic insects. London: Academic Press; 1981.

6. Krasnov BR. Functional and evolutionary ecology of fleas: a model for ecological parasitology. Cambridge: Cambridge University Press; 2008. p. 45-67.

7. Benoit JB, Denlinger DL. Meeting the challenges of on-host and off-host water balance in blood-feeding arthropods. J Insect Physiol. 2010;56:1366-76

8. Moore SL, Wilson K. Parasites as a viability cost of sexual selection in natural populations of mammals. Science. 2002;297:2015-8.

9. Klein SL. Hormonal and immunological mechanisms mediating sex differences in parasite infection. Parasite Immunol. 2004;26:247-64.

10. Zuk M, McKean KA. Sex differences in parasite infections: patterns and processes. Int J Parasitol. 1996;26:1009-24.

11. Hillegass MA, Waterman JM, Roth JD. Parasite removal increases reproductive success in a social African ground squirrel. Behav Ecol. 2010;21:696-700.

12. Altizer S, Dobson A, Hosseini P, Hudson P, Pascual M, Rohani P. Seasonality and the dynamics of infectious diseases. Ecol Lett. 2006;9:467-84.

13. Cattadori IM, Boag B, Bjørnstad ON, Cornell SJ, Hudson PJ. Peak shift and epidemiology in a seasonal host-nematode system. Proc R Soc Lond B Biol Sci. 2005:272:1163-9.

14. Alexander RD. The evolution of social behaviour. Ann Rev Ecol Syst. 1974;5:325-83.

15. Côté IM, Poulin R. Parasitism and group size in social animals: a metaanalysis. Behav Ecol. 1995;6:159-65.

16. Wilson K, Knell R, Boots M, Koch-Osborne J. Group living and investment in immune defence: an interspecific analysis. J Anim Ecol. 2003:72:133-43.

17. Bordes F, Blumstein DT, Morand S. Rodent sociality and parasite diversity. Bio Lett. 2007:3:692-4.

18. Krasnov BR, Shenbrot Gl, Khokhlova IS, Degen AA. Flea species richness and parameters of host body, host geography and host 'milieu.'J Anim Ecol. 2004;73:1121-8.

19. Mooring MS, Hart BL. Animal grouping for protection from parasites: selfish herd and encounter-dilution effects. Behaviour. 1992;123:173-93.

20. Hart DW, Medger K, van Jaarsveld B, Bennett NC. Is the Mahali mole-rat (Cryptomys hottentotus mahali) a spontaneous or induced ovulator? Can J Zool. 2020;98(5):299-305.

21. Moolman M, Bennett NC, Schoeman AS. The social structure and dominance hierarchy of the highveld mole-rat Cryptomys hottentotus pretoriae (Rodentia: Bathyergidae). J Zool. 1998;246:193-201.

22. van Rensburg $\sqcup$, Bennett NC, van der Merwe M, Schoeman AS. Seasonal reproduction in the highveld mole-rat, Cryptomys hottentotus pretoriae (Rodentia: Bathyergidae). Can J Zool. 2002;80:810-20.
23. Hart BL. Behavioral adaptations to pathogens and parasites: five strategies. Neuro Biobehav Rev. 1990;14:273-94.

24. Sichilima AM, Bennett NC, Faulkes CG, Le Comber SC. Evolution of African mole-rat sociality: burrow architecture, rainfall and foraging in colonies of the cooperatively breeding Fukomys mechowii. J Zool. 2008;275:276-82

25. Bishop JM, Jarvis JU, Spinks AC, Bennett NC, Oryan C. Molecular insight into patterns of colony composition and paternity in the common molerat Cryptomys hottentotus hottentotus. Mol Ecol. 2004;13:1217-29.

26. van Rensburg $\sqcup$, Bennett NC, Van der Merwe M, Schoeman AS, Brinders J. Are non-reproductive male highveld mole-rats, Cryptomys hottentotus pretoriae physiologically suppressed while in the confines of the natal colony? J Zool. 2003;260:73-8.

27. Matthee S, Krasnov BR. Searching for generality in the patterns of parasite abundance and distribution: ectoparasites of a South African rodent Rhabdomys pumilio. Int J Parasitol. 2009;39:781-8.

28. Scharff A, Burda H, Tenora F, Kawalika M, Barus V. Parasites in social subterranean Zambian mole-rats (Cryptomys spp., Bathyergidae, Rodentia). J Zool. 1997:241:571-7.

29. Tenora F, Barus V, Prokes M, Sumbera R, Koubkova B. Helminths parasitizing the silvery mole-rat, Heliophobius argenteocinereus (Rodentia: Bathyergidae) from Malawi. Helminthologia. 2003;40:153-60.

30. Viljoen $\mathrm{H}$, Bennett NC, Ueckermann EA, Lutermann $\mathrm{H}$. The role of host traits, season and group size on parasite burdens in a cooperative mammal. PLoS ONE. 2011;6:e27003.

31. Henry LG, McKeever SA. Modification of the washing technique for quantitative evaluation of the ectoparasite load of small mammals. J Med Entomol. 1971:8:504-5.

32. Krantz GW, Walter ED. A manual of acarology. 3rd ed. Lubbock: Texas Tech University Press; 2009.

33. Segerman J. Siphonatera of Southern Africa. A handbook for the identification of fleas. Johannesburg: South African Institute for Medical Research; 1995.

34. Till WM. Ethiopian mites of the genus Androlaelaps Berlese s. lat. (Acari: Mesostigmata). London: British Museum (Natural History); 1963.

35. Bush AO, Lafferty KD, Lotz JM, Shostak AW. Parasitology meets ecology on its own terms: Margolis et al. revisited. J Parasitol. 1997;83:575-83.

36. Archer EK, Bennett NC, Faulkes CG, Lutermann H. Digging for answers: contributions of density- and frequency-dependent factors on ectoparasite burden in a social mammal. Popul Ecol. 2016;180:429-38.

37. Archer EK, Bennett NC, Ueckermann EA, Lutermann H. Ectoparasite burdens of the common mole-rat (Cryptomys hottentotus hottentotus) from the Cape Provinces of South Africa. J Parasitol. 2014;100:79-84.

38. De Graaff G. On the mole-rat (Cryptomys hottentotus damarensis) (Rodentia) in the Kalahari Gemsbok National Park. Koedoe. 1972;15:25-35.

39. De Graaff $G$. The rodents of Southern Africa: notes on their identification, distribution, ecology, and taxonomy. Pretoria: Butterworth-Heinemann; 1981.

\section{Publisher's Note}

Springer Nature remains neutral with regard to jurisdictional claims in published maps and institutional affiliations.

Ready to submit your research? Choose BMC and benefit from

- fast, convenient online submission

- thorough peer review by experienced researchers in your field

- rapid publication on acceptance

- support for research data, including large and complex data types

- gold Open Access which fosters wider collaboration and increased citations

- maximum visibility for your research: over 100M website views per year

At BMC, research is always in progress.

Learn more biomedcentral.com/submissions 